\title{
PHARMACEUTICAL GRANULATOR MACHINE USING ETHERNET MODBUS PROTOCOL AND LAB VIEW
}

\author{
Tandel Mayureshkumar B ${ }^{1}$, Ashish G Patel ${ }^{2}$ \\ ${ }^{I}$ M.Tech Scholar, Dept. of IC, Dharamsinh Desai University, Nadiad, Gujarat, India \\ ${ }^{2}$ Assistant Professor, Dept. of IC, Dharamsinh Desai University, Nadiad, Gujarat, India
}

\begin{abstract}
Industrial Automation have remained area of interest and focus globally since its rapid growth have started signifying its strength for real-life applications and have attracted researchers attention worldwide. Pharmaceutical Granulator Machine incorporates various technologies at different hierarchical levels for automation of Granulating process. This PG Paper puts forth Laboratory Ethernet Modbus Interfacing of mimic with PLC and Lab View HMI and Results of Pharmaceutical Granulator Machine using Ethernet MODBUS Protocol and Lab VIEW in manual mode. The work included detailed design and MODBUS Interfacing of the system for process industries. MODBUS-compliant PLC is wired to RJ 45, which is connected to a PC, based HMI. Thereafter, a simple, but effective model for the proposed system has been prepared for Demonstration purpose. MODBUS is selected because of interoperability, vendor independence and similar other relative advantages have been tested with model for Demonstration purpose which has been one of the major contribution of the work presented in the Dissertation. Various functionalities of the proposed system have been validated in laboratory.
\end{abstract}

Keywords: Automation, Human-Machine Interface Design, MODBUS, Virtual Instrumentation

\section{INTRODUCTION}

Pharmaceutical Granulator Machine (PGM) is a Supervisory Control and Data Acquisition (SCADA) system that incorporates various functionalities provided by the manual and automatic control system of a Granulating Process. PGM is a computerized, intelligent network of electronic devices, designed to monitor and control the Granulating process for creating fine granules, safety, security, information, and Alarm facilities. PGM maintains the manual and automatic operation of Electrical AC motors with specific materials used for batch-wise mixing, processes. It controls different motor speed operation. It continuously monitors these parameter values and also provides their settings by manual and automatic intervention. The Pharmaceutical Granulating System (PGS) controlled by automation (implemented using Programmable Logic Controller (PLC) is often referred to as Pharmaceutical Granulator Machine. PGM saves upon material consumption and thereby, optimizes operational and maintenance costs as compared to an uncontrolled PGS. This paper presents a design of PGM based on wired MODBUS protocols. Although not formally standardized, MODBUS is regarded as an open protocol. The forthcoming sections present detailed design of the proposed PGM for typical industrial application, including design objectives, functionalities, motor speed operation and Lab view based HMI of the system. The design has been validated by implementing most of the functionalities of the proposed system in the laboratory implementation. The paper ends with the concluding notes and identifying future scope of work in form of suggestions for further value addition.

\section{SYSTEM DESCRIPTIONS}

\subsection{Design Objectives}

Following are the design objectives of proposed PGM:

- To handle Manual and Automatic Operation

- Simple and user-friendly Human-Machine Interface (HMI).

- Low cost of the solution.

- Simplicity, future expandability and interoperability.

\subsection{Functionalities}

Typically, the functionalities such as communication and information exchange need high data rates, while the features like Automation, safety needs low data rates but low latency, high network reliability and data security [6]. Moreover, the designs of PGM for process industries must be cost-effective and easy to operate without specialized training. The proposed system has been designed to implement the functionalities mentioned below:

- $\quad$ Reporting alarms in the HMI.

- $\quad$ Provides control of field controllers via HMI.

- $\quad$ Security or Interlock provided by HMI.

- $\quad$ Speed control provided via HMI

\subsection{Design of PGM}

The proposed PGM design has been validated by most of its functionalities in laboratory. The proposed PGM has threelayer modular design architecture and modules.

- Mimic

- Modbus Interfacing

- Lab View HMI 
The proposed PGM system is designed and implemented to handle Industry grade LED's of Pharmaceutical Granulator Machine (mimic) as a case study for experimental validation and testing of the prototype resulted as an outcome of the proposed design of Granulating Process. Fig -1: given below shows the Block diagram of the typical PGM.PLC with LAB View HMI which provides controlling and monitoring for Pharmaceutical Granulator Machine. By giving commands from Lab View HMI we can operate PLC and Pharmaceutical Granulator System. The proposed PGM design having Alarm facility. The proposed system includes panel mount LED indicator and speaker as a major hardware. Panel mount LED indicator shows the contactors, chopper, impeller and crowl motor as well as hooter. In proposed system we are also placed switches which is work as input and shows the faults coming from the granulator systems such faults are impeller overload and impeller thermostat, chopper over load and chopper thermostat, crowl overload and crowl thermostat, emergency stop.

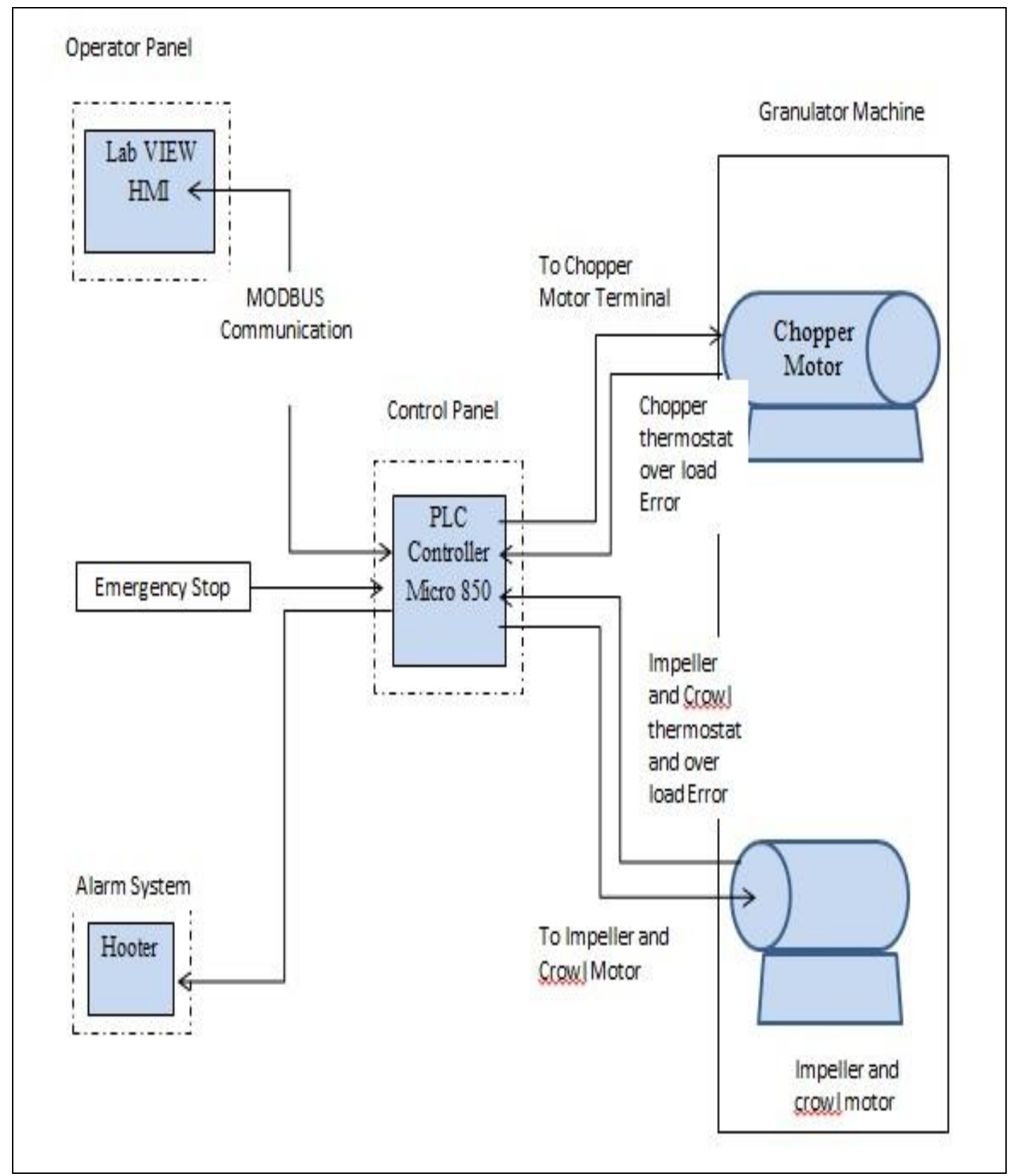

Fig -1: Block diagram of typical PGM

Alarm subsystem makes necessary provisions for critical alarm annunciation. Audio-visual annunciation is generated for the faults configured or operational errors, such as Impeller error, Chopper error, etc. Data communication between the PLC controller and the corresponding HMI is established through RJ 45 based MODBUS communication. The input to the controller is interfaced with computer running Lab VIEW HMI. Main HMI is used for monitoring and control in a typical MODBUS network. 


\subsection{Motor Speed Operation}

Here, in this proposed PGM system impeller motor, crowl motor and chopper motor are present which simply induction motors. From these motors chopper and impeller motors are start through star delta starter method and crowl motor is starting through direct on line starter method. In PGM system crowl motor is work with impeller motor and made three speed operation crowl, slow and fast speed. Here, crowl motor is used to give clutch for impeller motor. Impeller motor is used for mixing pharmaceutical powder and made it fine. Chopper motor work alone and it having two speed operations slow and fast speed. Chopper motor is used as here bowl is big so chopper motor is work as secondary impeller. Fig -2: shows speed operation flow of chopper, impeller and crowl motors. Here, impeller star and impeller fast contactors indicate impeller fast operation, for chopper motor, chopper star and chopper fast indicate chopper fast operation. Chopper motor is work in off to slow, slow to fast, fast to slow and slow or fast to off speed operation and impeller motor with crowl motor work in off to crowl, crowl to slow, crowl to fast, slow to crowl, slow to fast, and crowl or slow or fast to off speed operation. For crowl to fast motor operation motors are first go to slow operation.

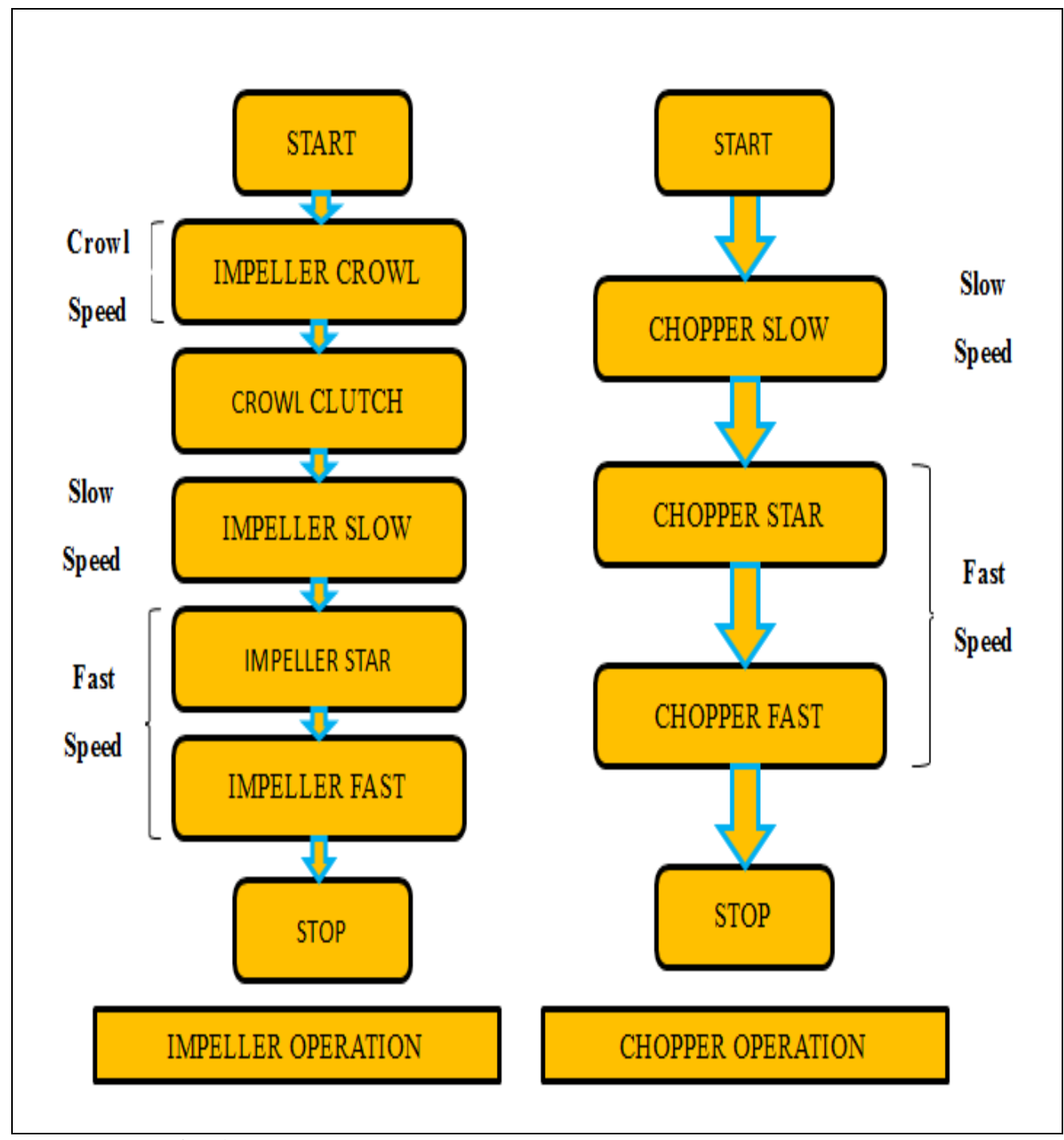

Fig -2: speed operation flow of chopper, impeller and crowl motor

\subsection{About HMI}

The application software for the PGM is developed using Lab VIEW platform. It can be operated in two modes:

- Automatic Control mode

- Manual Control mode

For HMI development, we have used Laboratory Virtual Instrumentation Engineering Workbench (Lab VIEW), which is highlevel graphical programming language developed by National Instruments. The HMI prepared using Lab VIEW helps to give a command to PLC. Fig -3: shows the operator VI which having proposed system controls and Alarms indicators 


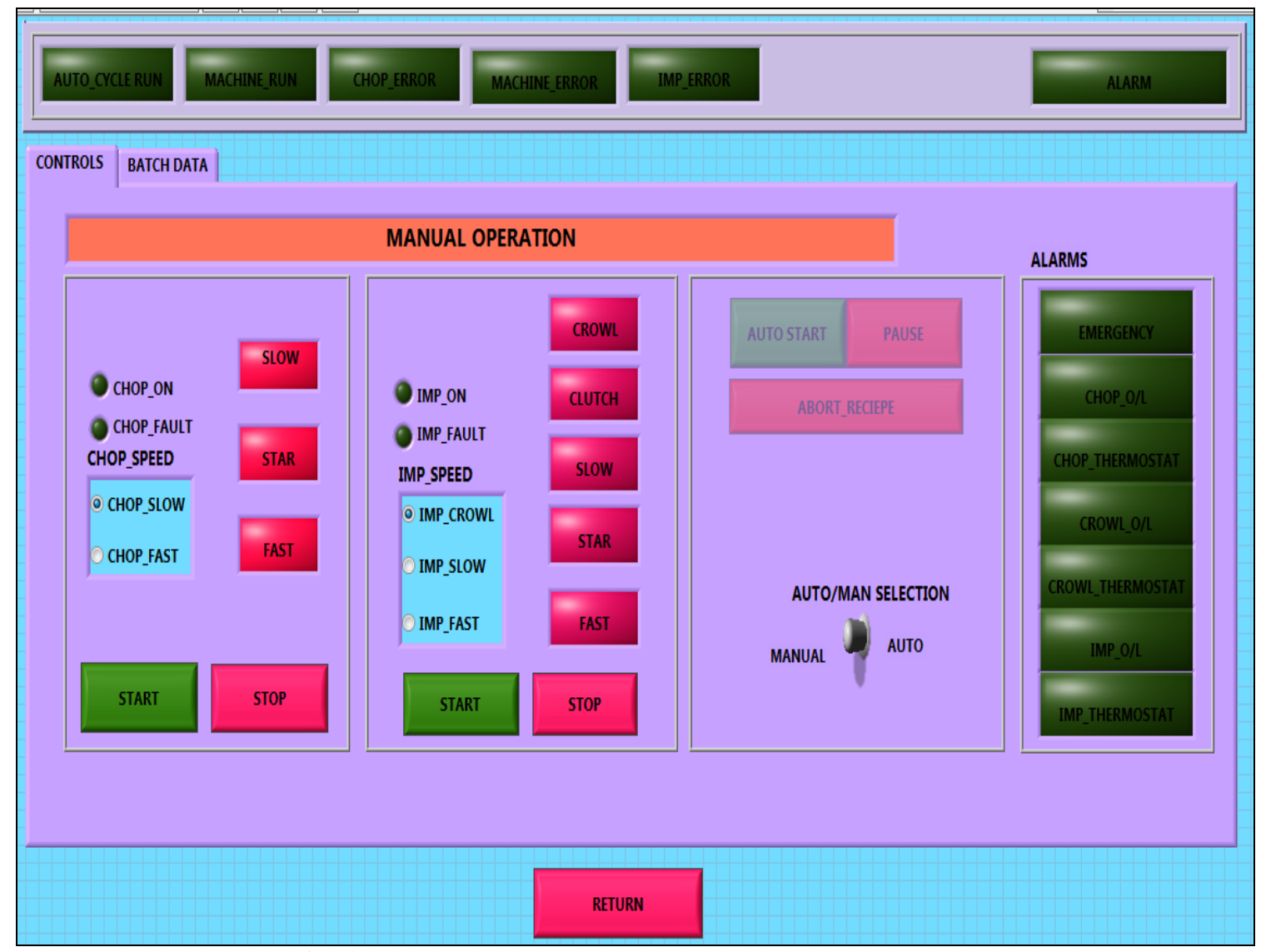

Fig -3: Operator VI which having proposed system controls

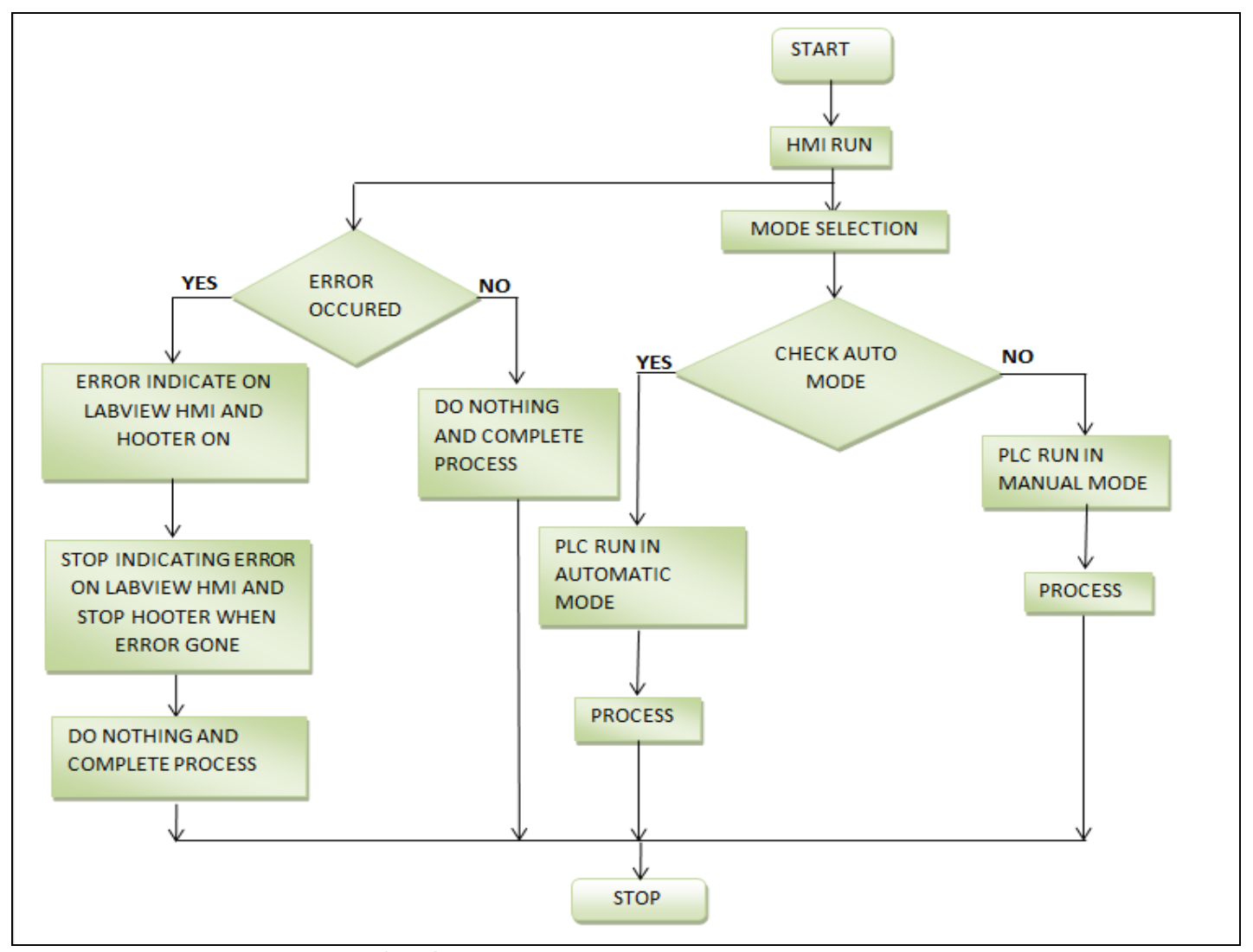

Fig -4: Mode selection in Proposed System

Fig -4: shows the Mode selection in proposed system. It also gives the information of errors during automatic or manual operation. 


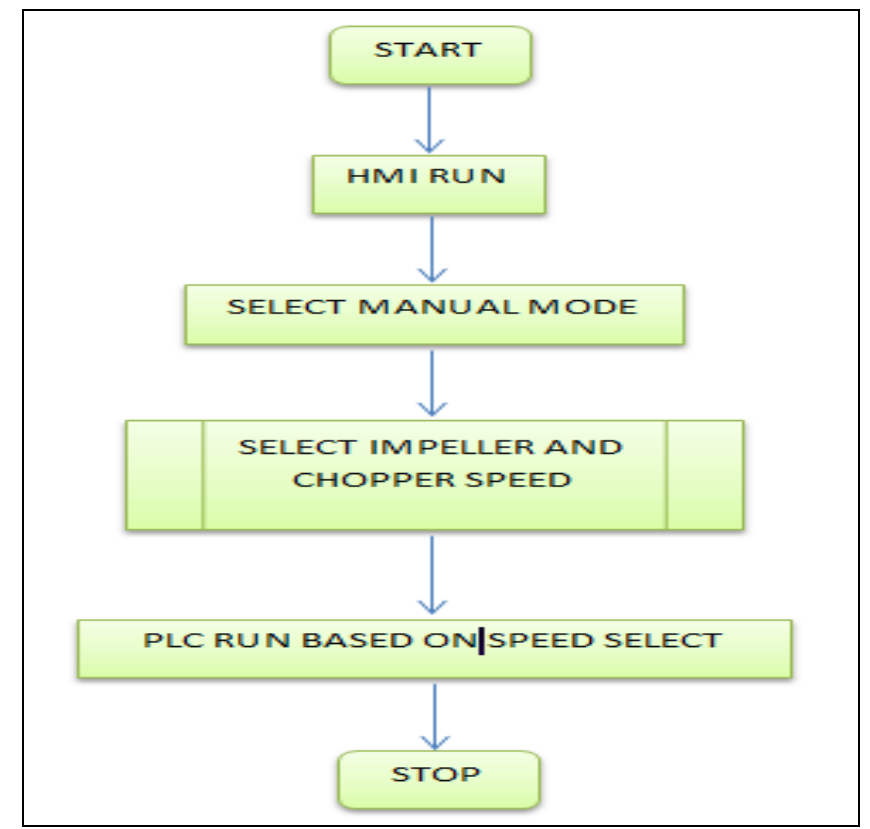

Fig -5: Manual Control Mode

Fig -5: shows the flow chart of the Manual control mode of the proposed system design. It gives the detail information about the Manual control mode of proposed system. Fig -5: shows the detail flow of operation.

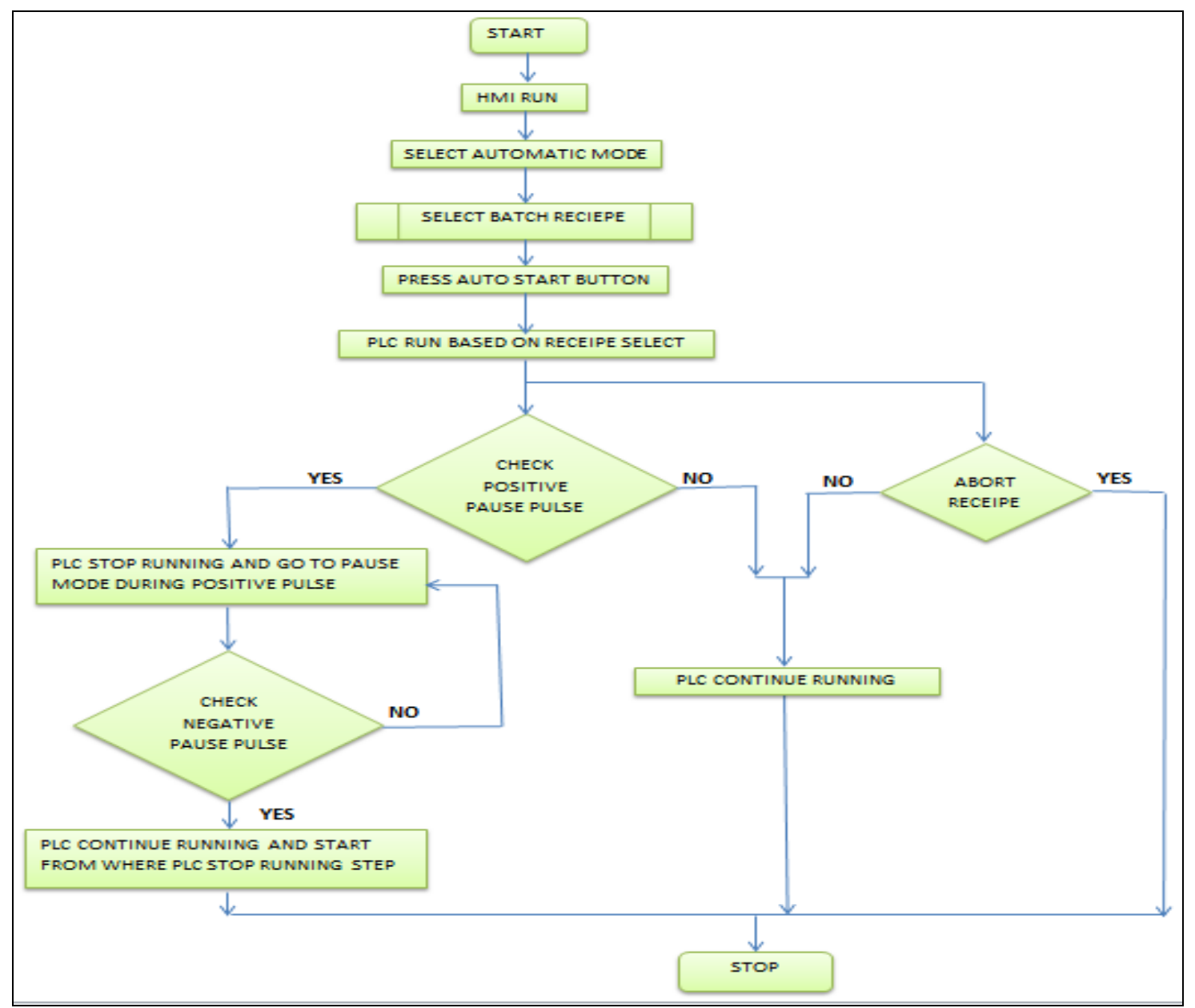

Fig -6: Automatic Control Mode

Fig -6: shows the flow chart of the Automatic Control Mode of the proposed system. It gives the detail information about the Automatic operation of proposed system. It also gives the information about the Pause, Abort Recipe button functionality. 


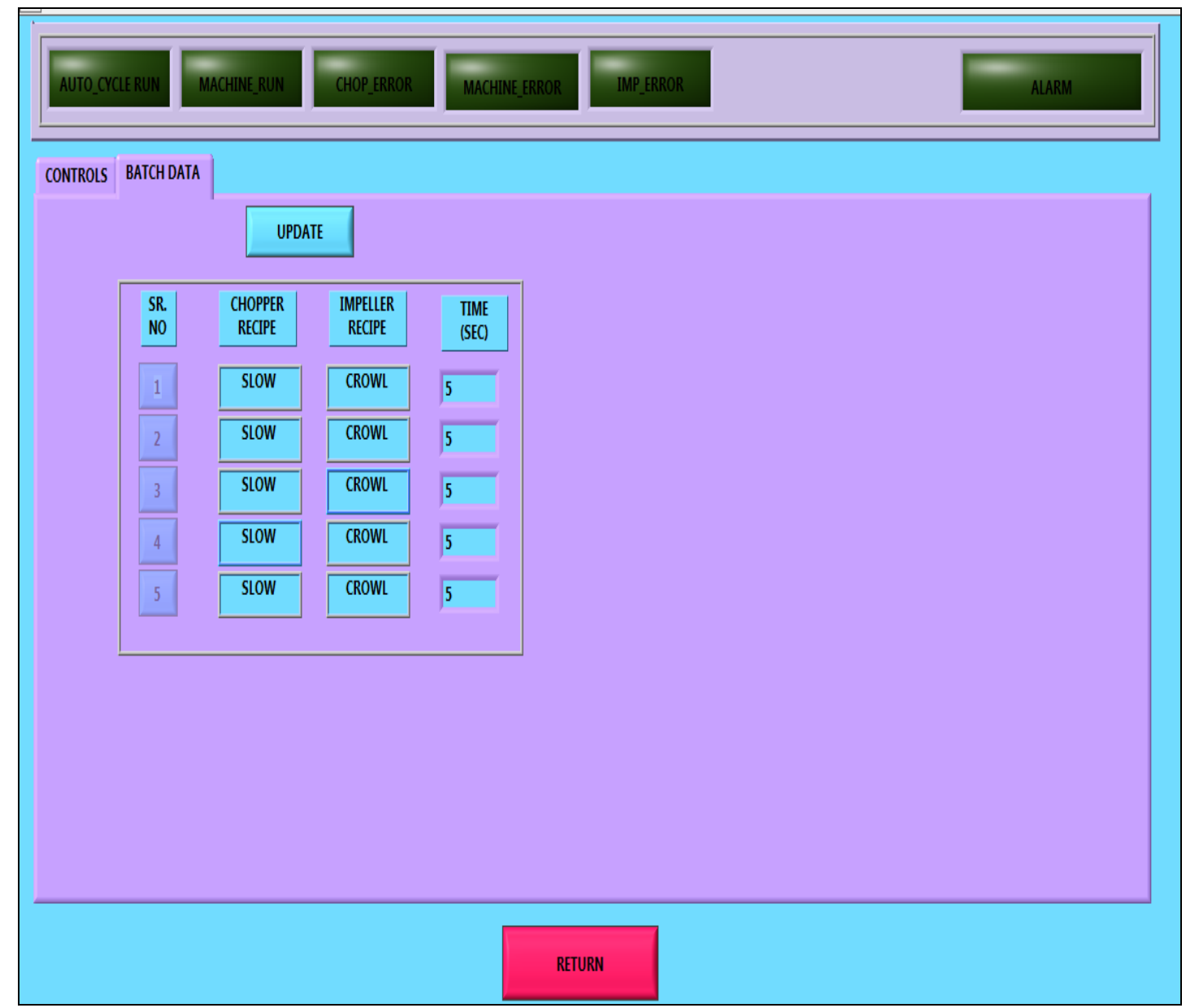

Fig -7: Recipe selection

Recipe is the set of step by step chopper speed and Impeller speed operation. Fig -7: shows the recipe selection. By pressing UPDATE button we can send the recipe to the PLC and according to that PLC run in Automatic mode.

\section{MODBUS COMMUNICATION WITH PLC}

\section{AND LABVIEW HMI}

We can communicate Lab View HMI and PLC with the MODBUS Protocol simply follows the following steps

STEP 1: Make a PLC Program in CCW (Connected Component Workbench).

STEP 2: Do an Internet Protocol Setting, Ethernet Port setting and Modbus Mapping in CCW

STEP 3: Load Program in PLC

STEP 4: Make a Lab View HMI using MODBUS Toolkit and Internet Toolkit.

STEP 5: Connect PLC and Computer with Registered Jack 45

STEP 6: See the Results

\section{RESULTS AND DISCUSSION}

The proposed system is capable to present the results of PLC Micro 850 Interfacing of Granulator System in a Manual Mode using Ethernet MODBUS Protocol and Lab View HMI as shown in Fig -8:.In Fig -8: ON Panel mount
LEDs Indicates that contactors are $\mathrm{ON}$ of the star delta connection which drive the Chopper motor in slow and fast speed and impeller motor in crowl, slow and fast with crowl Motor and same result is shown in Lab View HMI and PLC board as well as on mimic of granulator system. 


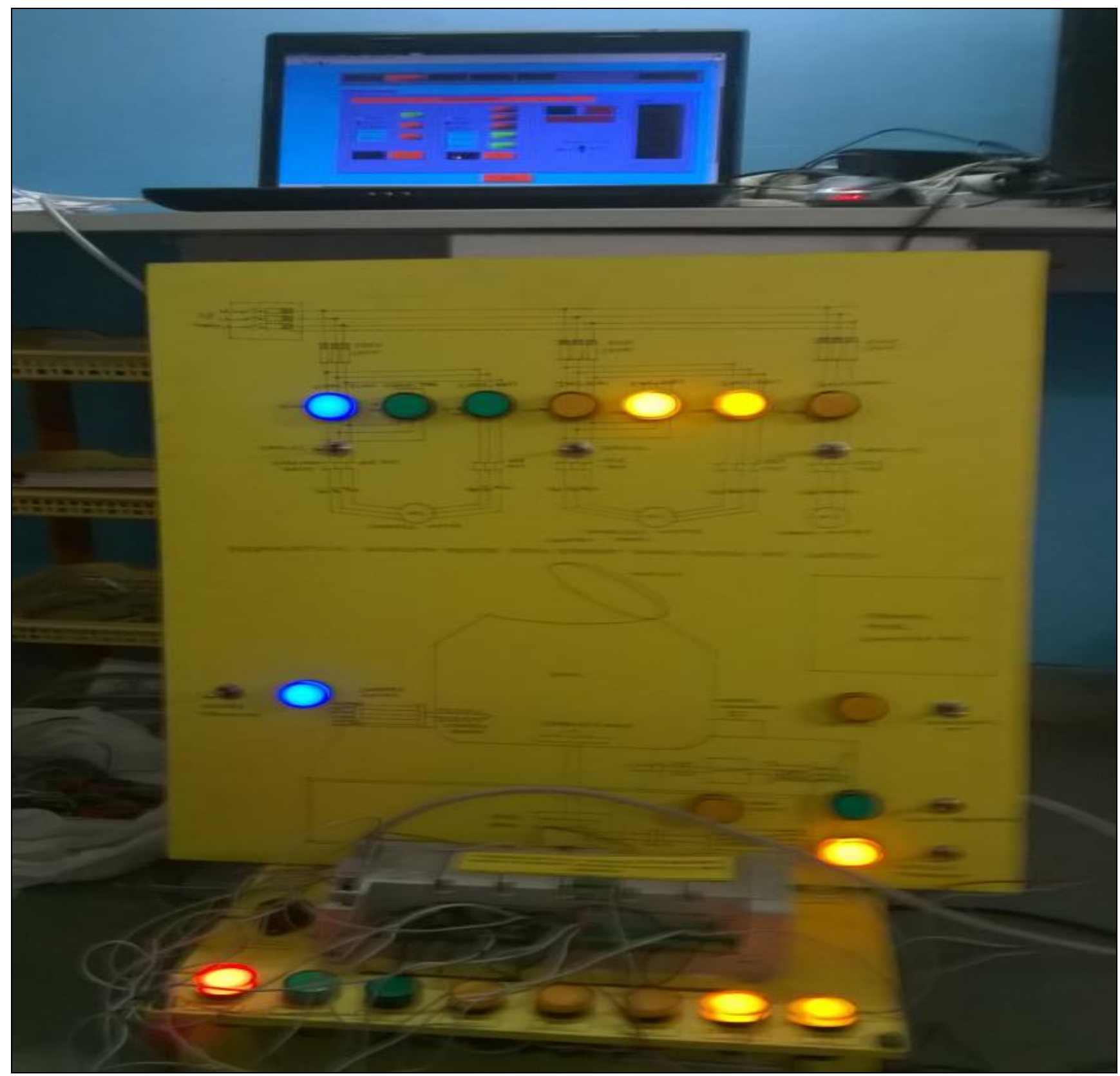

Fig -8: Computer running Lab View HMI with PLC Hardware

Fig -8: show the computer running Lab VIEW HMI connected to PLC Hardware

\section{CONCLUSION}

The design tested in laboratory establishing MODBUS communication between Mimic and the computer running HMI through RJ 45. In this design, applications are tested in Manual mode using Lab VIEW HMIs. MODBUS is selected because of interoperability, vendor independence and similar other relative advantages. MODBUS protocol is used for the time-critical process-alarm data exchange for high speed client-server communication. Automation based on vendor independent automation protocols such as MODBUS as well as HMI development technology using Lab VIEW platform results into fully scalable, reliable and future expandable cost-effective solution for automation.

\section{FUTURE SCOPES}

This work can be further extended by

- Giving Automatic Operation mode and Error detection to the Pharmaceutical granulator machine.

- $\quad$ Adding more and more functionalities to the HMI to make them more informative and user-friendly.

\section{ACKNOWLEDGEMENTS}

The first author thankfully acknowledges the support and co-operation provided by Dharmsinh Desai University, Nadiad, Gujarat, India and $\mathrm{M} / \mathrm{s}$ Vbtech Automation, Ahmedabad, Gujarat, India in development of this work. 


\section{REFERENCES}

[1] Anajli S. Ashtekar, Bhagsen J. Parvat, Chandrakant B. Kadu, "Application of MODBUS to Communicate the PLC and Lab VIEW for Real Time Process Control", International Journal of Emerging Science and Engineering (IJESE) ISSN: 2319-6378, Volume-1, Issue-11, September 2013

[2] "Modbus application protocol specifications v1.1b3" April 26, 2012

[3] "Modbus TCP unplugged" An introduction to Modbus TCP networking prepared by Real time automation

[4] J.-D. Decotignie, "Ethernet-Based Real-Time and Industrial Communications", Proc. of the IEEE, vol. 93, no. 6, 2005.

[5] P. Yadav, J.S.Chauhan, P.Kannojia, N.K Jain, V.Tomar" A review : On Scale-up Factor Determination of Rapid Mixer Granulator", Scholar Research Library ISSN:0975-8071, USA CODE N : DPLKB4

[6] Sunil D Patel, Jignesh G Bhatt, Himanshu G Bhavsar" Design and Implementation of RS485/MODBUS based Automatic Batch Weighing System using Lab VIEW"

\section{BIOGRAPHIES}

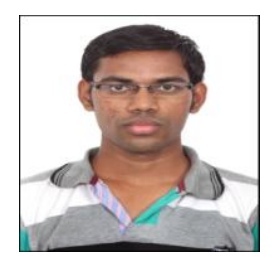

Mayureshkumar B Tandel received his Bachelor of Engineering with Electronics and Communication Engineering from $\mathrm{C} \quad \mathrm{K}$ Pithawalla College of Engineering and Technology, Surat, Gujarat Technological University, Ahmedabad, Gujarat, India. His areas of interests include Automation Systems, Virtual Instrumentation and Industrial Process Control, Biomedical Instrumentation, Artificial Intelligence and Robotics, Nano Technology.

E-Mail: er.mayureshkumar@gmail.com

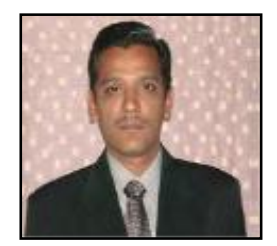

Prof. Ashish G. Patel received his Bachelor of Engineering with Instrumentation and Control Engineering from College Dharmsinh Desai University, Nadiad in 1999 and Master of Engineering with Instrumentation and Control Engineering from Dharmsinh Desai University, Nadiad in 2004. He has 4.5 year industrial experience and more than 9 year teaching experience. His area of interest in the various fields of instrumentation like Analytical Instrumentation (Electron Microscopy), Biomedical Instrumentation, Distributed control System, Robotics \& Control systems etc.

E Mail: ashishddit@gmail.com 